\title{
Análisis Comparativo de la Repetibilidad y Reproductibilidad de Dos Métodos de Medición de la Dimensión Vertical en Rehabilitación Oral: Una Revisión Sistemática
}

\author{
Comparative Analysis of the Repeatability and Reproducibility of Two Methods for \\ Measuring the Vertical Dimension in Oral Rehabilitation: A Systematic Review
}

\begin{abstract}
Lorena Brenes Ortega*; Gorka Santamaría Arrieta*; Felipe J. Fernández-González ${ }^{\star * *}$; Nerea Martín Blanco ${ }^{\star \star * *}$; José

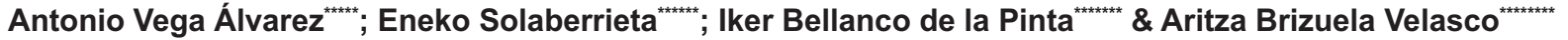

BRENES, O. L.; SANTAMARÍA, A. G.; FERNÁNDEZ-GONZÁLEZ, F. J.; MARTÍN, B. N.; VEGA, Á. J. A.; SOLABERRIETA, E.; BELLANCO DE LA PINTA, I. \& BRIZUELA, V. A. Análisis comparativo de la repetibilidad y reproductibilidad de dos métodos de medición de la dimensión vertical en rehabilitación oral: una revisión sistemática. Int. J. Odontostomat., 10(1):55-62, 2016.

RESUMEN: El objetivo de este estudio establecer si el método de evaluación de la dimensión vertical mediante cefalometría y el convencional con rodillos de articulación, consiguen resultados sin diferencias estadísticamente significativas. Realización de una revisión sistemática conforme a la metodología PICO del Centro de Medicina Basada en la Evidencia de Oxford. Se formula una pregunta dirigida al objetivo fijado y se elabora una estrategia de búsqueda empleando MeSH key words específicos derivados de la pregunta en la base de datos PubMed hasta 2015 y libros de rehabilitación oral. Se consultan un total de 833 artículos por título, siendo 26 revisados a texto completo. Se analizan trabajos sobre distintos métodos de determinación de la dimensión vertical, haciendo especial hincapié en los basados en métodos cefalométricos. Finalmente 9 artículos cumplen los criterios de inclusión y se realiza una evaluación crítica del nivel de evidencia. No se ha encontrado suficiente evidencia científica que demuestre la repetibilidad de ambos métodos. Es necesario realizar estudios controlados y aleatorizados que comparen ambos métodos para determinar si son repetibles y reproducibles y obtienen resultados sin diferencias estadísticamente significativas.

PALABRAS CLAVE: dimensión vertical, rehabilitación oral, rodillos de articulación, cefalometría.

\section{INTRODUCCIÓN}

La determinación de la dimensión vertical (DV) es una etapa crítica en el plan de tratamiento del paciente, en rehabilitación oral (Quiroga Del Pozo et al., 2012). El Glosario de términos de Prostodoncia (The glossary of prosthodontic terms, 2005) define la DV como la distancia establecida entre dos puntos, situa- dos normalmente en la raíz nasal y en el margen del mentón, haciendo referencia a la posición de la mandíbula con respecto al maxilar en el plano vertical. La distancia obtenida con los dientes en oclusión, indica la dimensión vertical de oclusión (DVO), mientras que la dimensión vertical de reposo (DVR), se consigue

\footnotetext{
Graduada en Odontología, Universidad del País Vasco UPV-EHU, Leioa, España.

* Profesor del Postgrado de Especialista Universitario en Implantología oral. Departamento de Estomatología I. Universidad del País Vasco UPV-EHU, Leioa, España.

*** Departamento de Cirugía y Especialidades Médicas. Universidad de Oviedo, Oviedo, España.

.*** Profesora del Postgrado de Especialista Universitario en Implantología oral. Departamento de Estomatología I. Universidad del País Vasco UPV-EHU, Leioa, España.

***** Facultad de Ciencias de la Salud. Universidad Autónoma de Chile, Temucom, Chile.

*mor** Departamento de Expresión Gráfica e Ingeniería de Proyectos. Universidad del País Vasco UPV/EHU, Leioa, España.

*turtur Alumno del Postgrado de Especialista Universitario en Implantología oral. Departamento de Estomatología I. Universidad del País Vasco UPV-EHU, Leioa, España.

Profesor Colaborador de Honor del Departamento de Cirugía y Especialidades Médico Quirúrgicas. Prótesis y Oclusión. Universidad de Oviedo, Oviedo, España
} 
BRENES, O. L.; SANTAMARÍA, A. G.; FERnÁNDEZ-GonZÁLEZ, F. J.; MARTíN, B. N.; VEGA, Á. J. A.; SOLABERRIETA, E.; BELLANCO DE LA PINTA, I. \& BRIZUELA, V. A. Análisis comparativo de la repetibilidad y reproductibilidad de dos métodos de medición de la dimensión vertical en rehabilitación oral: una revisión sistemática. Int. J. Odontostomat., 10(1):55-62, 2016.

con la mandíbula relajada, sin contacto dentario, siendo la diferencia entre ambas el espacio libre interoclusal (Alonso et al., 1999).

Variaciones en la DV son percibidas como una alteración estética, pero también se han asociado con problemas funcionales durante la masticación, la deglución, la pronunciación fonética e incluso en la respiración (Huumonen et al., 2010). Estas variaciones pueden ser la base etiopatogénica de ciertos cuadros patológicos, caracterizados por dolores de cabeza y de la articulación temporomandibular (ATM), dolores musculares, tinnitus, mareos, vértigos, entre otros, incluso lesiones en las mucosa oral o perioral (Ramírez et al., 2013). Estos síntomas pueden aparecer, de manera iatrogénica, durante el manejo de la DV en rehabilitación, aunque algunos autores citan que son autolimitados y transitorios, resolviéndose entre 1-2 semanas (Abduo, 2012; Discacciati et al., 2013). Así mismo, otros autores discuten la relación entre los trastornos temporomandibulares y la DV reducida (Abduo \& Lyons, 2012).

No obstante, la ATM junto con los dientes forma una articulación que debe estar en armonía (Alonso et al.) y es por ello que durante la rehabilitación oral resulta imprescindible manejar correctamente la DV para conseguir una correcta función, estética y un control de la ATM para prevenir los problemas anteriormente citados. La mayoría de los métodos aplicados a tal fin han sido desarrollados para la determinación en pacientes totalmente edéntulos. Un aumento de DV en pacientes parcialmente edéntulos debe considerar factores como la estructura del diente residual, el espacio disponible para la restauración y la estética oclusal, estando raramente indicado un incremento de más de $5 \mathrm{~mm}$ (Abduo \& Lyons).

El método más empleado es el uso de rodillos articulares cuyo registro se basa en una serie de pruebas estáticas y dinámicas (Capusselli \& Schvartz, 1987). También se han empleado otros métodos, como por ejemplo correlacionar la DVO con diferentes medidas antropométricas (Ladda et al., 2013), el análisis mediante el craneómetro de Knebelman (Quiroga Del Pozo et al.), la kinesiografía y registro de silicona en la medición del espacio más cercano del habla (Rizzatti et al., 2007), la relación entre los dientes anteriores y la reflexión mucolabial (Fayz et al., 1987), la distancia ojo-oído en la predicción de la distancia nariz-mentón (Chou et al., 1994) e incluso se han desarrollado métodos que determinen la DV y la posición del plano oclusal de forma simultánea (Koller et al., 1992). Pese a la variedad de métodos empleados, Fayz \& Eslami (1988) sostienen que no existe uno completamente exacto y no se encuentran ventajas significativas entre ellos, salvo las de coste, tiempo y equipos empleados. Finalmente, han valorado el uso del análisis cefalométrico como un estudio de bajo coste y radiación, pudiendo establecer la orientación del plano oclusal, inclinación de las piezas anteriores y guía anterior, teniendo como referencia tejidos duros y no blandos (Carrera Vidal et al., 2010).

El objetivo del presente trabajo es establecer si ambos métodos de evaluación de la DV (cefalométrico y convencional mediante rodillos articulares) consiguen los mismos resultados sin diferencias estadísticamente significativas.

\section{MATERIAL Y MÉTODO}

Con el fin de responder al objetivo marcado, se realiza una revisión sistemática de la literatura conforme a la sistemática PICO (Patient or Population, Intervention, Control or Comparison y Outcome) del CEBM (Centre for Evidence-Based Medicine) de la Universidad de Oxford.

Formulación de la pregunta dirigida: ¿Se consiguen los mismos resultados a través de los métodos cefalométricos y convencionales en el restablecimiento de la dimensión vertical en rehabilitación oral? Se emplean MeSH key words específicos en la base de datos PubMed, dirigidos a responder a la pregunta PICO. La Tabla I contiene los detalles de la sistemática utilizada. La estrategia de búsqueda se diseña para obtener un número más alto de entradas relacionadas, sin restricciones, salvo para el término "Vertical Dimension of Occlusion" que se acota a los últimos años (2012-2015). Finalmente se realiza la tipificación del nivel de la prueba de los estudios incluidos y su grado de recomendación en base a la clasificación del CEBM de Oxford.

\section{RESULTADOS}

Los artículos incluidos en la revisión se obtienen mediante la estrategia de búsqueda que muestra la Tabla II. La búsqueda inicial obtuvo un total de 833 entradas. Se realizó una selección por título, por resumen y finalmente se revisaron a texto completo un to- 
BRENES, O. L.; SANTAMARÍA, A. G.; FERNÁNDEZ-GONZÁLEZ, F. J.; MARTíN, B. N.; VEGA, Á. J. A.; SOLABERRIETA, E.; BELLANCO DE LA PINTA, I. \& BRIZUELA, V. A. Análisis comparativo de la repetibilidad y reproductibilidad de dos métodos de medición de la dimensión vertical en rehabilitación oral: una revisión sistemática. Int. J. Odontostomat., 10(1):55-62, 2016.

Tabla I. Detalles de la sistemática utilizada para la búsqueda bibliográfica.

\begin{tabular}{ll}
\hline Sistemática de búsqueda sistematizada \\
\hline Pregunta dirigida: ¿Se consiguen los mismos resultados a través de los métodos ce falométricos y \\
convencionales en el restablecimiento de la dimensión vertical en rehabilitación oral? \\
PICO (Patient or Population, Intervention, Control or Comparison y Outcome) \\
$\begin{array}{l}\text { Problema } \\
\text { Intervención }\end{array}$ \\
Reestablecer la dimensión vertical \\
Outcome & Rehabilitación oral \\
Bases de datos & Análisis cefalométrico Vs Rodillos de articulación \\
Libros & Repetibilidad de los resultados \\
Criterios de inclusión & Pubmed \\
& Tratamiento del desdentado total. Ed. Mundi, Oclusión y Diagnóstico en \\
& Rehabilitación Oral. Ed. Panamericana \\
& Artículos disponibles en inglés y castellano, Metaanálisis de estudios controlados y \\
& aleatorizados que comparen ambos métodos, Estudios clínicos controlados y \\
aleatorizados, Estudios clínicos retrospectivos y prospectivos. Estudios observacionales \\
del cálculo de la D V en pacientes dentados y que muestren los criterios de inclusión \\
(dentición natural con topes oclusales posteriores, ausencia de síntomas subjetivos de \\
patología de la ATM). Revisiones sistemáticas. Las prótesis evaluadas en los estudios \\
debían ser realizadas mediante método convencional o cefalométrico.
\end{tabular}

tal de 26 trabajos. Mediante los criterios de inclusión establecidos (Tabla II), sólo 7 artículos pudieron ser incluidos. Posteriormente, a partir de las referencias de los artículos disponibles, se seleccionaron 2 trabajos y finalmente la revisión sistemática incluye un total 9 artículos.

Se incluyen 4 estudios de investigación teórica (Orthlieb et al., 2000; Strajnic et al., 2008; Tavano et al., 2012; Yamashita et al., 2015), 2 estudios de investigación clínica (Bassi et al., 2001a; Brzoza et al., 2005), 1 estudio de cohorte de baja calidad (Ciftci et al., 2005), 1 de casos y controles de baja calidad (Unger, 1990) y 1 estudio ecológico (Carrera Vidal et al.).

La Tabla III muestra la tipificación del nivel de la prueba y el grado de recomendación de los estudios incluidos en base a la clasificación del CEBM de
Oxford. No es posible realizar el análisis de los riesgos de sesgo basados en la herramienta Cochrane ya que no se ha encontrado ningún estudio controlado y aleatorizado en la revisión. Los trabajos incluidos basados en el estudio cefalométrico, aplican diferentes tipos de análisis y parámetros relacionados con la DVO, por lo que existe heterogeneidad en cuanto a su diseño.

Todos los estudios que analizan pacientes dentados excluyen aquellos que hayan sido sometidos a tratamientos de ortodoncia, cirugía o que presenten grandes restauraciones. Otros criterios como enfermedades sistémicas, alteraciones temporomandibulares o ausencia de dientes fueron los más señalados. En cuanto a los pacientes edéntulos incluidos, la mayoría de los trabajos indican que debían serlo durante al menos 5 años pero no más de 15 .

Tabla II. Resultados búsqueda inicial.

\begin{tabular}{lcc}
\hline Palabras clave & \multicolumn{2}{c}{ Pubmed } \\
Incluidos
\end{tabular}


BRENES, O. L.; SANTAMARÍA, A. G.; FERNÁNDEZ-GONZÁLEZ, F. J.; MARTíN, B. N.; VEGA, Á. J. A.; SOLABERRIETA, E.; BELLANCO DE LA PINTA, I. \& BRIZUELA, V. A. Análisis comparativo de la repetibilidad y reproductibilidad de dos métodos de medición de la dimensión vertical en rehabilitación oral: una revisión sistemática. Int. J. Odontostomat., 10(1):55-62, 2016.

Tabla III. Tipificación del nivel de la prueba y Grado de Recomendación de los trabajos incluidos en base al CEBM de Oxford.

\begin{tabular}{llcc}
\hline Estudio & Tipo de Estudio & $\begin{array}{c}\text { Nivel de Evidencia } \\
\text { CEBM Oxford }\end{array}$ & $\begin{array}{c}\text { Grado de } \\
\text { Recomendación }\end{array}$ \\
\hline Tavano et al. (2012) & Investigación teórica & 5 & $\mathrm{D}$ \\
Bassi et al. (2001) & Investigación clínica & 2c & $\mathrm{B}$ \\
Brzoza et al. (2005) & Investigación clínica & $2 \mathrm{c}$ & $\mathrm{B}$ \\
Çiftçi et al. (2005) & Cohorte de baja calidad & $2 \mathrm{~b}$ & $\mathrm{~B}$ \\
Strajnic et al. (2008) & Investigación teórica & 5 & $\mathrm{D}$ \\
Unger (1990) & Casos y controles de baja calidad & 4 & $\mathrm{C}$ \\
Yamashita et al. (2014) & Investigación teórica & 5 & $\mathrm{D}$ \\
Carrera et al. (2010) & Estudio ecológico & $2 \mathrm{c}$ & $\mathrm{B}$ \\
Orthlieb et al. $(2000)$ & Investigación teórica & 5 & $\mathrm{D}$ \\
\hline
\end{tabular}

Se ha considerado reposicionar la mandíbula en el plano vertical en pacientes que hayan sufrido pérdida de DV mediante una correlación de ángulos (Fig. 1) a partir de la posición fija del maxilar en el cráneo (Tavano et al.). Otros estudios analizan la significación estadística entre la forma de la mandíbula y la altura facial inferior en oclusión (Orthlieb et al.) siendo el ángulo goniaco el parámetro que mayor coeficiente de correlación obtiene (Fig. 2).

Yamashita et al., obtienen una fórmula para predecir la DVO usando parámetros lineales y angu-

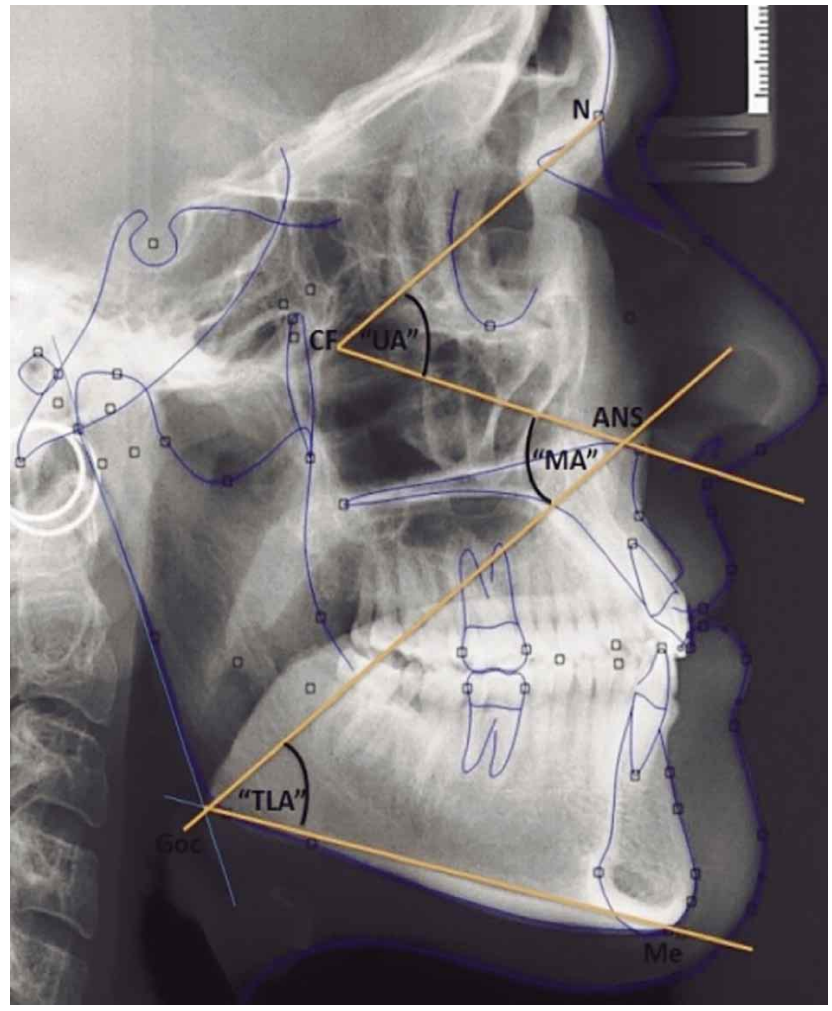

Fig.1. Análisis Seraidarian-Tavano mediante correlación de ángulos. lares de análisis cefalométricos, siendo válida para un rango entre $37^{\circ}-59^{\circ}$, por lo que es aplicable en los pacientes en los que su valor se desvíe considerablemente de la media.

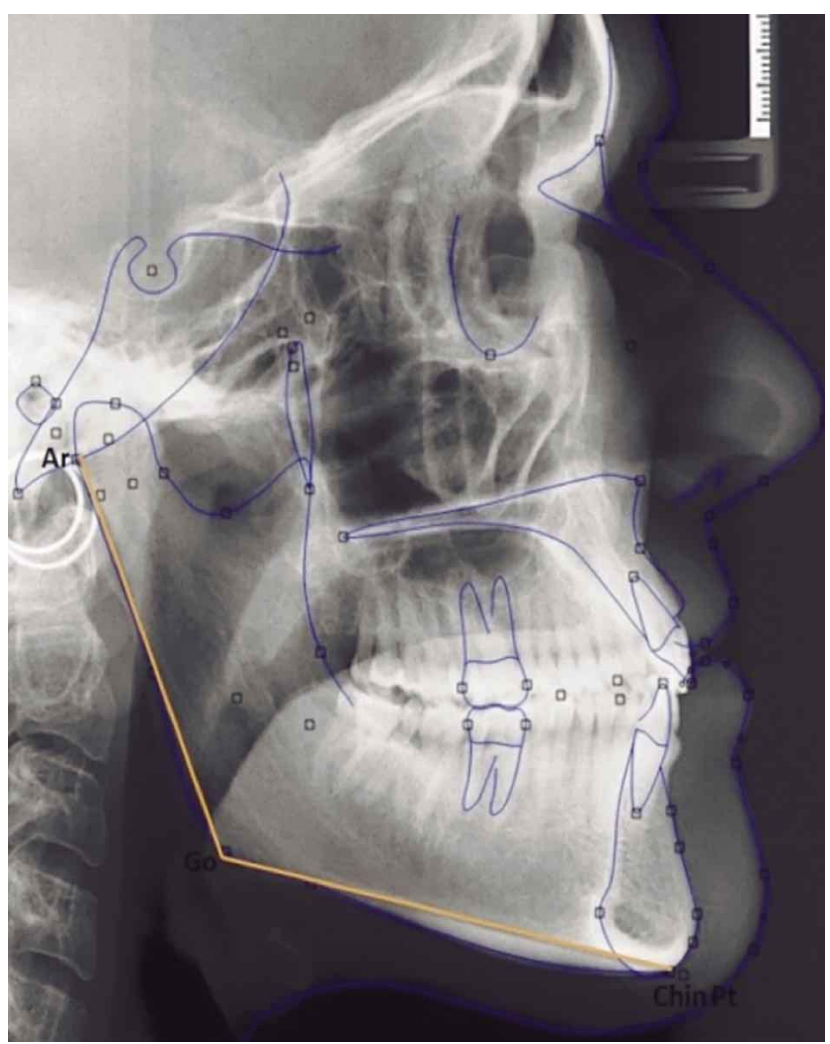

Fig. 2. Ángulo Goniaco

La distancia cefalométrica N-Gn (Nasion Gnation) (Fig. 3) con una media de $122,7 \mathrm{~mm}$ es considerada como otra alternativa para el control en la medición de la DV (Unger) o la proporción entre la altura facial superior e inferior (N-ANS:ANS-Me) (Fig. 4) también podría usarse para tener un control durante la fabricación de las prótesis (Strajnic et al.). 


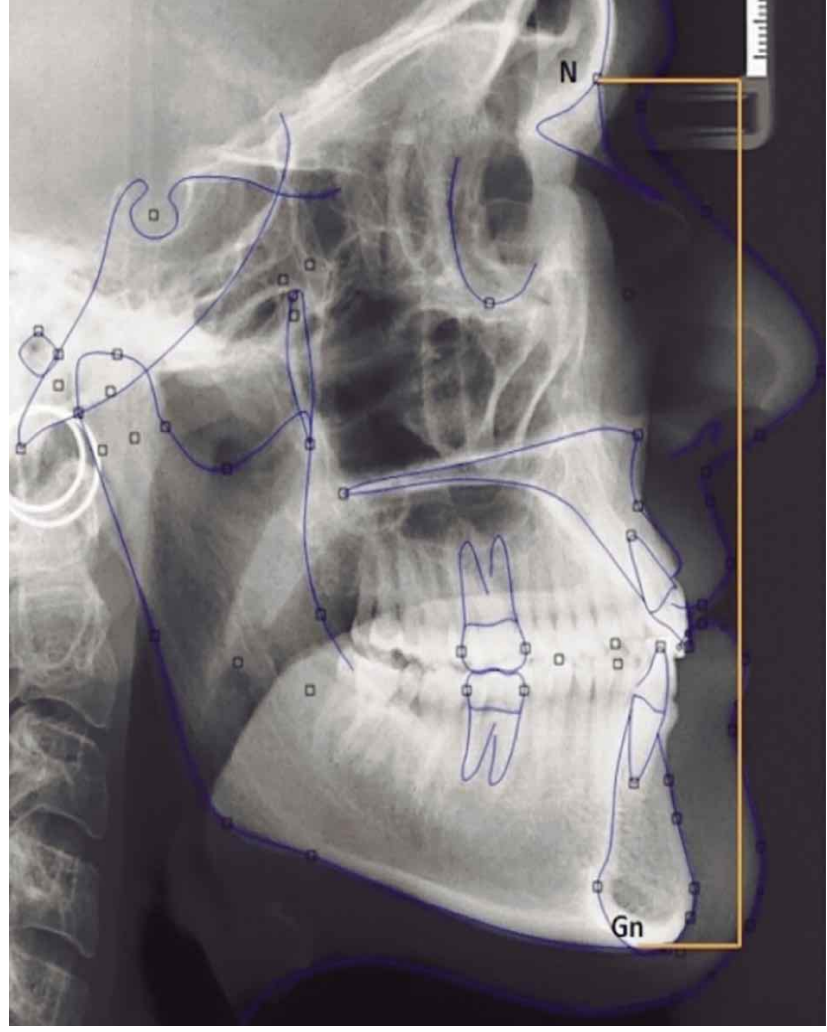

Fig. 3. Distancia N-Gn.

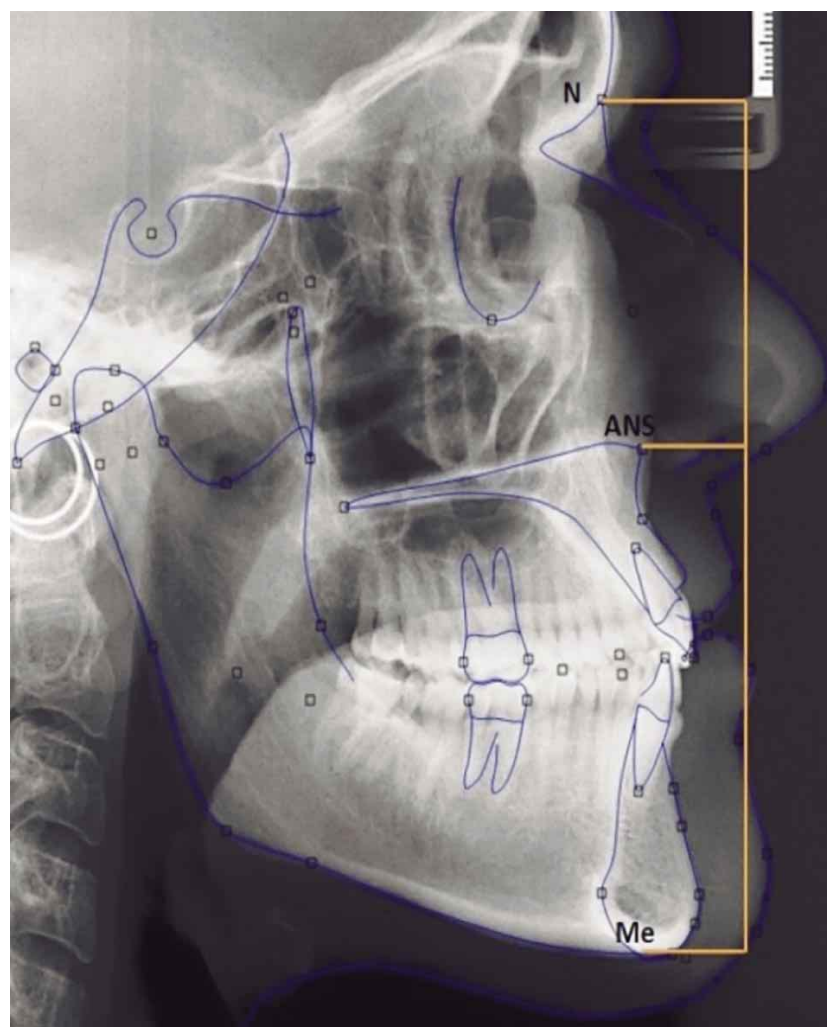

Fig. 4. Distancia N-ANS y ANS-Me.
Ciftci et al., establecen que el ángulo de la altura facial inferior, el ángulo formado por el plano mandibular y Frankfort y el ángulo GoGn-SN son los principales determinantes de la DVO ya que aumentan significativamente al corregir la DVO mediante nuevas prótesis (Fig. 5). Sin embargo, variaciones de $1 \mathrm{~mm}$ en la DVO no muestran diferencias estadísticamente significativas en relación al ángulo de la altura inferior (Fig. 5) (Carrera Vidal et al.).

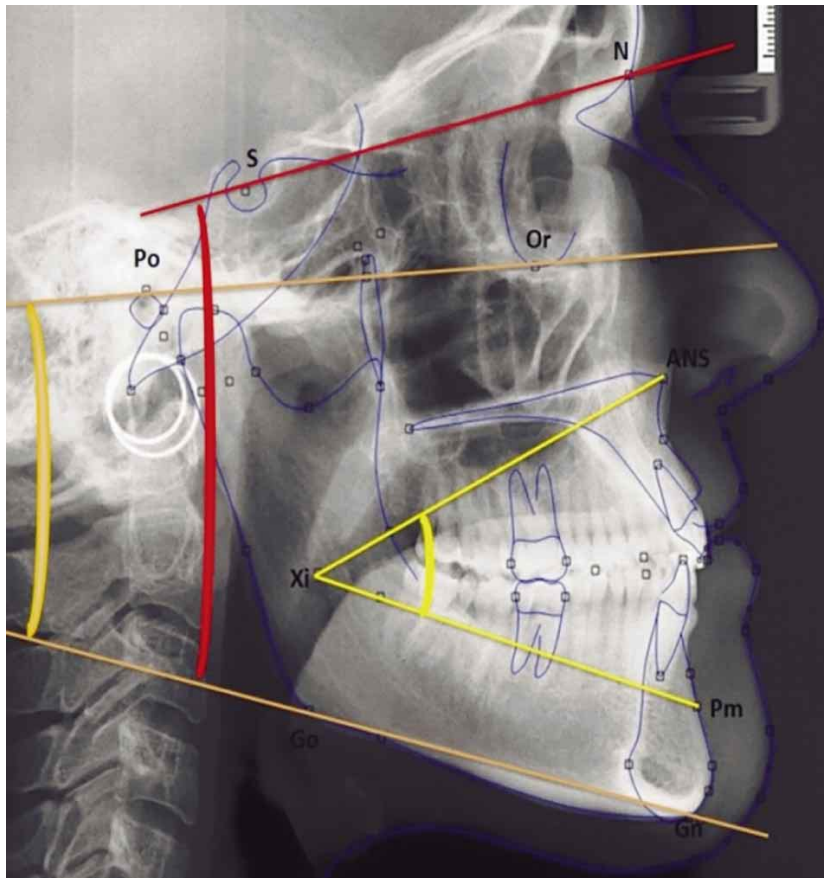

Fig. 5. Plano SN, Plano de Frankfort (PoOr), Plano mandibular (GoGn), Ángulo altura facial inferior (Xi-ANS-Pm).

Cuando se evalúa cefalométricamente a individuos edéntulos cuyas dentaduras completas han sido construidas basándose en parámetros clínicos mediante rodillos articulares, los resultados muestran que los parámetros clínicos obtienen una DV consistentemente menor, una anterorrotación del plano oclusal y un predominio de la arcada superior en la subdivisión del espacio de la dentadura (Bassi et al., 2001b).

Brzoza et al., observan que las mediciones cefalométricas en pacientes edéntulos con sus prótesis puestas eran similares a las medidas cuando se toman las radiografías sin ellas, correspondiéndose tanto las medidas realizadas en tejidos duros como en tejidos blandos. En base a estos resultados, realizan prótesis en dos pacientes usando las medidas de la cefalometría sin prótesis para ajustar los rodillos, y la nueva radiografía muestra que la predicción del rodillo era similar a la prótesis existente. 
BRENES, O. L.; SANTAMARÍA, A. G.; FERNÁNDEZ-GONZÁLEZ, F. J.; MARTíN, B. N.; VEGA, Á. J. A.; SOLABERRIETA, E.; BELLANCO DE LA PINTA, I. \& BRIZUELA, V. A. Análisis comparativo de la repetibilidad y reproductibilidad de dos métodos de medición de la dimensión vertical en rehabilitación oral: una revisión sistemática. Int. J. Odontostomat., 10(1):55-62, 2016.

\section{DISCUSIÓN}

El objetivo del trabajo era determinar si el método de medición de la DV a través del análisis cefalométrico conseguía los mismos resultados que el convencional sin diferencias estadísticamente significativas.

Algunos estudios consideran que el análisis de la morfología ósea es más preciso que otros métodos para valorar la DVO. Ricketts (1981) aporta información sobre parámetros cefalométricos de manera que la forma de la mandíbula puede medirse a través del ángulo mandibular (goniaco) y el arco mandibular. La altura facial inferior de Ricketts es capaz de detectar cambios cuando se realizan variaciones en la DV (Carossa et al., 1990; Unger; Ciftci et al.; Carrera Vidal et al.; Zielak et al., 2014) y permite evaluar si el prognatismo mandibular que presentan las personas que llevan prótesis durante mucho tiempo es debido a una pérdida de DV o a que el paciente tiene una clase III ya que aumenta al insertar la prótesis nueva (Ciftci et al.).

El ángulo goniaco es el que mayor coeficiente de correlación alcanza en relación a la DV (Orthlieb et al.; Yamashita et al.), sin embargo, se piensa que está relacionado con la forma y función de los músculos masticatorios y que va cambiando con la edad. Estudios en radiografías panorámicas han mostrado que el edentulismo y el género está asociado con un ángulo mandibular mayor y la altura condilar y de la rama mandibular es más pequeña que en los pacientes dentados (Huumonen et al.), por lo que puede no ser adecuado utilizar el ángulo goniaco en la predicción de la DVO.

Algunos autores sugieren que las distintas razas presentan diferencias en la morfología ósea. Por ello han intentado desarrollar fórmulas utilizando parámetros lineales y angulares y de esta forma la predicción de la DV sería más exacta que aplicar un valor promedio fijo para todos (Yamashita et al.), sin embargo, el rango de valores obtenido es amplio, por lo que promediar a todos los pacientes en base a estos resultados puede ser inexacto. Además, la muestra se compone de sujetos de un sólo grupo racial (japoneses), indicativo de que la variabilidad entre ellos probablemente sea muy pequeña.

Por otro lado, cuando se intenta comparar los métodos convencionales y los cefalométricos, no se encuentra una relación clara respecto al incremento de cera en los rodillos y los resultados cefalométricos. Zielak et al., asumen que cada grado del ángulo altura inferior de Ricketts corresponde a $1 \mathrm{~mm}$ de la altura de los rodillos, sin embargo, Carrera Vidal et al., cuando realizan aumentos de $1 \mathrm{~mm}$, la menor variación que se observa es de $0,5^{\circ}$ y la mayor es de $2^{\circ}$. Los resultados indican que las variaciones del ángulo no son de igual magnitud en todos los individuos cuando se incrementa la misma distancia, ya que dependerá de la forma mandibular y la posterorrotación producida.

Las mediciones de la DV mediante marcas en la piel del método convencional parecen no ser fiables. Resultados de estudios muestran que aumentos de la DV se corresponden con la nueva distancia medida entre marcas óseas, mientras que sólo un número limitado de pacientes muestran correlación positiva con las marcas de la piel (Carossa et al.). Bassi et al. (2001a) sospechan que la diferencia entre los resultados al evaluar cefalométricamente dentaduras completas realizadas por método convencional puede deberse a que el método clínico está basado en la función para lograr la estabilidad de la prótesis y los datos cefalométricos hacen referencia a la morfología ósea basada en sujetos dentados.

Brzoza et al. justifican la coincidencia entre las mediciones de las radiografías (con y sin dentadura) añadiendo que el sistema neuromuscular se mantiene cuando la pérdida de dientes es reciente. Esta correlación no existe en otros estudios incluidos en la revisión; la mayoría muestran un ángulo altura facial inferior disminuido y al aumentar la DVO, este aumenta también. Por este motivo, la predicción de los rodillos tal y como se realiza en este trabajo no puede extrapolarse a aquellas cefalometrías en las que la altura facial inferior esté disminuida.

En general, los estudios consideran que el método cefalométrico es más preciso y permite un control de la DVO a través del método convencional previamente realizado (Orthlieb et al.; Ciftci et al.; Strajnic et al.; Zielak et al.). No obstante, los estudios cefalométricos son muy heterogéneos y ninguno de ellos está perfectamente descrito ni estandarizado. Sus niveles de la prueba son bajos y no evalúan lo más importante, que ambos métodos deben tener como objetivo conseguir una DV que sea eficiente con la estética, función, que respete la ATM y que el resultado debe ser controlado durante un periodo de seguimiento adecuado. 
BRENES, O. L.; SANTAMARÍA, A. G.; FERNÁNDEZ-GONZÁLEZ, F. J.; MARTíN, B. N.; VEGA, Á. J. A.; SOLABERRIETA, E.; BELLANCO DE LA PINTA, I. \& BRIZUELA, V. A. Análisis comparativo de la repetibilidad y reproductibilidad de dos métodos de medición de la dimensión vertical en rehabilitación oral: una revisión sistemática. Int. J. Odontostomat., 10(1):55-62, 2016.

\section{CONCLUSIONES}

No hay suficiente evidencia que demuestre la repetibilidad de ambos métodos. Pese a estas limitaciones, cuando se comparan ambos métodos (convencional y cefalométrico), la diferencia no parece ser estadísticamente significativa.
Es necesario realizar estudios aleatorizados y controlados comparando ambos métodos para determinar si son estadísticamente repetibles y reproducibles y en base a los resultados, proponer cuál resulta más eficiente.

BRENES, O. L.; SANTAMARÍA, A. G.; FERNÁNDEZ-GONZÁLEZ, F. J.; MARTÍN, B. N.; VEGA, Á. J. A.; SOLABERRIETA, E.; BELLANCO DE LA PINTA, I. \& BRIZUELA, V. A. Comparative analysis of the repeatability and reproducibility of two methods for measuring the vertical dimension in oral rehabilitation: a systematic review. Int. J. Odontostomat., 10(1):55-62, 2016.

ABSTRACT: The objective of this work was to establish if the method of evaluation of the vertical dimension by cephalometry and the common one with joint rollers, obtains results without statistically significant differences. We carried out a systematic review based on the PICO methodology of the Center for Evidence-Based Medicine for Oxford. A question aimed at the objective was made, and a search strategy was developed using specific MeSH key words derived from the question in the Pubmed database up to 2015 and oral rehabilitation books. A total of 833 items were consulted by tittle, and for 26 of these full text was reviewed. Works on different methods for determining the vertical dimension were studied, with special emphasis on those based on cephalometric methods. Finally 9 articles fulfilled the criteria for inclusion and a critical assessment of the evidence level was performed. We did not find sufficient scientific evidence to demonstrate the repeatability of these methods. It is necessary to realize randomized controlled trials comparing both methods to determine if they were statistically repeatable and reproducible.

KEY WORDS: vertical dimension, oral rehabilitation, joint rollers, cephalometry.

\section{REFERENCIAS BIBLIOGRÁFICAS}

Abduo, J. Safety of increasing vertical dimension of occlusion: a systematic review. Quintessence Int., 43(5):369-80, 2012.

Abduo, J. \& Lyons, K. Clinical considerations for increasing occlusal vertical dimension: a review. Aust. Dent. J., 57(1):2-10, 2012.

Alonso, A. A.; Albertini, J. S. \& Bechelli, A. H. Oclusión y Diagnóstico en Rehabilitación Oral. Buenos Aires, Médica Panamericana, 1999.

Bassi, F.; Deregibus, A.; Previgliano, V.; Bracco, P. \& Preti, G. Evaluation of the utility of cephalometric parameters in constructing complete denture. Part I: placement of posterior teeth. J. Oral Rehabil., 28(3):234-8, 2001a.

Bassi, F.; Rizzatti, A.; Schierano, G. \& Preti, G. Evaluation of the utility of cephalometric parameters in constructing complete denture. Part II: placement of anterior teeth. $J$. Oral Rehabil., 28(4):349-53, 2001b.

Brzoza, D.; Barrera, N.; Contasti, G. \& Hernández, A. Predicting vertical dimension with cephalograms, for edentulous patients. Gerodontology, 22(2):98-103, 2005.

Capusselli, H. O. \& Schvartz, T. Tratamiento del Desdentado Total. $3^{a}$ ed. Buenos Aires, Mundi, 1987.
Carossa, S.; Catapano, S.; Scotti, R. \& Preti, G. The unreliability of facial measurements in the determination of the vertical dimension of occlusion in edentulous patients. J. Oral Rehabil., 17(3):287-90, 1990.

Carrera Vidal, C.; Larrucea Verdugo, C. \& Galaz Valdés, C. Detection of increases of Vertical Occlusal Dimension by Ricketts cephalometric analysis. Rev. Clin. Periodoncia Implantol. Rehabil. Oral, 3(2):79-85, 2010.

Chou, T. M.; Moore, D. J.; Young, L. Jr. \& Glaros, A. G. A diagnostic craniometric method for determining occlusal vertical dimension. J. Prosthet. Dent., 71(6):568-74, 1994.

Ciftci, Y.; Kocadereli, I.; Canay, S. \& Senyilmaz, P. Cephalometric evaluation of maxillomandibular relationships in patients wearing complete dentures: a pilot study. Angle Orthod., 75(5):821-5, 2005.

Discacciati, J. A.; Lemos de Souza, E.; Vasconcellos, W. A.; Costa, S. C. \& Barros, Vde. M. Increased vertical dimension of occlusion: signs, symptoms, diagnosis, treatment and options. J. Contemp. Dent. Pract., 14(1):123-8, 2013.

Fayz, F. \& Eslami, A. Determination of occlusal vertical dimension: a literature review. J. Prosthet. Dent., 59(3):321-3, 1988. 
BRENES, O. L.; SANTAMARÍA, A. G.; FERNÁNDEZ-GONZÁLEZ, F. J.; MARTín, B. N.; VEGA, Á. J. A.; SOLABERRIETA, E.; BELLANCO DE LA PINTA, I. \& BRIZUELA, V. A. Análisis

comparativo de la repetibilidad y reproductibilidad de dos métodos de medición de la dimensión vertical en rehabilitación oral: una revisión sistemática. Int. J. Odontostomat., 10(1):55-62, 2016.

Fayz, F.; Eslami, A. \& Graser, G. N. Use of anterior teeth measurements in determining occlusal vertical dimension. J. Prosthet. Dent., 58(3):317-22, 1987.

Huumonen, S.; Sipilä, K.; Haikola, B.; Tapio, M.; Söderholm, A. L.; Remes-Lyly, T.; Oikarinen, K. \& Raustia, A. M. Influence of edentulousness on gonial angle, ramus and condylar height. J. Oral Rehabil., 37(1):34-8, 2010.

Koller, M. M.; Merlini, L.; Spandre, G. \& Palla, S. A comparative study of two methods for the orientation of the occlusal plane and the determination of the vertical dimension of occlusion in edentulous patients. J. Oral Rehabil., 19(4):413-25, 1992.

Ladda, R.; Bhandari, A. J.; Kasat, V. O. \& Angadi, G. S. A new technique to determine vertical dimension of occlusion from anthropometric measurements of fingers. Indian J. Dent. Res., 24(3):316-20, 2013.

Orthlieb, J. D.; Laurent, M. \& Laplanche, O. Cephalometric estimation of vertical dimension of occlusion. J. Oral Rehabil., 27(9):802-7, 2000.

Quiroga Del Pozo, R.; Riquelme Belmar, R.; Sierra Fuentes, M.; Del Pozo Bassi, J. \& Quiroga-Aravena, R. Determinación de la Dimensión Vertical Oclusal en desdentados totales: comparación de métodos convencionales con el craneómetro de Knebelman. Rev. Clin. Periodoncia Implantol. Rehabil. Oral., 5(1):20-4, 2012.

Ramirez, L. M.; Echeverría, P.; Zea, F. J.; \& Ballesteros, L. E. Vertical dimension on edentulous patient: Relationship with symptoms reported. Int. J. Morphol., 31(2):672-80, 2013.

Rizzatti, A.; Ceruti, P.; Mussano, F.; Erovigni, F. \& Preti, G. A new clinical method for evaluating the closest speaking space in dentulous and edentulous subjects: a pilot study. Int. J. Prosthodont., 20(3):259-62, 2007.

Ricketts, R. M. Perspectives in the clinical application of cephalometrics. The first fifty years. Angle Orthod., 51(2):115-50, 1981.

Strajnic, L.; Stanisic-Sinobad, D.; Markovic, D. \& Stojanovic, L. Cephalometric indicators of the vertical dimension of occlusion. Coll. Antropol., 32(2):535-41, 2008.

Tavano, K. T.; Seraidarian, P. I.; de Oliveira, D. D. \& Jansen, W. C. Determination of vertical dimension of occlusion in dentate patients by cephalometric analysis--pilot study. Gerodontology, 29(2):e297-305, 2012.

The glossary of prosthodontic terms. J. Prosthet. Dent., 94(1):10-92, 2005.

Unger, J. W. Comparison of vertical morphologic measurements on dentulous and edentulous patients. J. Prosthet. Dent., 64(2):232-4, 1990.
Yamashita, S.; Shimizu, M. \& Katada, H. A newly proposed method to predict optimum occlusal vertical dimension. J. Prosthodont., 24(4):287-90, 2015.

Zielak, J. C.; Gulin Neto, D.; da Cunha, L. F.; Deliberador, T. M. \& Giovanini, A. F. Cephalometric approach to the occlusal vertical dimension reestablishment. Case Rep. Dent., 2014:920840, 2014.

Dirección de Correspondencia

Lorena Brenes Ortega

Parque de los Enamorados 5, 31014.

Pamplona

Navarra

ESPAÑA

Email: lorena_brenesortega@hotmail.com

Recibido: 26-11-2015

Aceptado: 11-03-2016 